\title{
Prevalence of Celiac Disease in Children and Adolescents With Inflammatory Bowel Disease
}

Ayman Eskander ${ }^{1}$, Omar I. Saadah ${ }^{2}$, Abdelrahman A. Abdelrazek ${ }^{3}$, Mahmoud Mosli ${ }^{4}$, Hadeel A. Alsufyani ${ }^{5}$, Safa Meshaal ${ }^{6}$, Amera M. Hasnoon ${ }^{1}$, Sara Tarek ${ }^{1}$, Abobakr Abdelgalil ${ }^{3}$

1. Pediatric Gastroenterology, Cairo University, Cairo, EGY 2. Pediatric Gastroenterology, King Abdulaziz University, Jeddah, SAU 3. Pediatrics, Cairo University, Cairo, EGY 4. Internal Medicine/Gastroenterology, King Abdulaziz University, Jeddah, SAU 5. Medical Physiology, King Abdulaziz University, Jeddah, SAU 6. Pathology, Cairo University, Cairo, EGY

Corresponding author: Omar I. Saadah, osaadah@kau.edu.sa

\section{Abstract}

\section{Introduction}

The association between inflammatory bowel disease (IBD) - particularly its two main subtypes, ulcerative colitis (UC) and Crohn's disease (CD) - and celiac disease (CeD) has been attributed to an overlap in the mechanism of immune dysregulation that characterizes these conditions. Owing to the paucity of studies that have explored this condition in pediatric patients, we examined the prevalence of $\mathrm{CeD}$ in children with IBD.

\section{Materials and methods}

This is a cross-sectional study of children aged two to 18 years with IBD that were diagnosed between 2016 and 2018. Clinical, demographic, laboratory, and endoscopic data were analysed. Serology for CeD measured the immunoglobulin A tissue transglutaminase (IgA-tTG) antibodies, and the diagnosis was confirmed histologically through small bowel biopsies.

\section{Results}

The study included 101 patients with IBD ( $83.2 \%$ with UC and $16.8 \%$ with CD). The mean age was $8.7 \pm 4.0$ years. Males constituted $59.4 \%$ of the cohort, and only $3 \%$ had perianal disease. Ileocolonic involvement was reported in $64.7 \%$ and non-stricturing and non-penetrating behaviour in $76.7 \%$ of $\mathrm{CD}$ patients. Pancolitis constituted $45.2 \%$ of UC patients. Ten patients ( $9.9 \%$ ) had positive serology based on IgA-tTG antibodies, three (approximately $3 \%$ ) had CeD based on biopsy findings, two patients (2\%) had CD, and one patient (1\%) had UC. Patients with confirmed CeD had a significantly higher frequency of symptoms of gaseous sensation and bloating $(\mathrm{P}=0.003)$ and abdominal distension $(\mathrm{P}=0.04)$.

Received 08/17/2020

Review began 08/18/2020 Review ended 08/19/2020 Published 08/23/2020

\section{○) Copyright 2020}

Eskander et al. This is an open access article distributed under the terms of the Creative Commons Attribution License CC-BY 4.0., which permits unrestricted use, distribution, and reproduction in any medium, provided the original author and source are credited.

\section{Conclusions}

The prevalence of CeD in Egyptian children with IBD is higher than previously reported in a number of similar studies. Abdominal bloating and gaseous sensation were identified as associated symptoms.

Categories: Pediatrics, Gastroenterology

Keywords: ulcerative colitis, crohn's disease, celiac disease, ibd, children, egypt

\section{Introduction}

Inflammatory bowel disease (IBD) comprises a group of inflammatory conditions which include Crohn's disease (CD) and ulcerative colitis (UC). CD can affect any site along the gastrointestinal tract, with inflammation that occurs in a skipped pattern, although with a transmural coverage, and can lead to bowel stricturing or fistulization. By contrast, within UC, inflammation is restricted to the colon and rectum and tends to be continuous [1]. Celiac disease (CeD) is an immune-related condition that can result in destruction of the intestinal mucosa of the small bowel as a result of inflammation triggered by exposure to gluten, as well as other environmental factors, in certain genetically predisposed individuals. The genetic susceptibility of CeD patients has been linked to human leukocyte antigen (HLA)-DQ2 and HLA-DQ8 haplotypes [2]. The etiology of IBD and CeD is likely multifactorial, as a result of a complex interaction between genetic and environmental factors. The dysregulation that occurs involves both the innate and adaptive immune pathways, and results in activation of the inflammatory cascade that results in gastrointestinal mucosal inflammation [3]. Both conditions may possibly have common genetic pathways, since they have four shared risk loci, interleukin 18 receptor accessory protein (IL18RAP), protein tyrosine phosphatase, non-receptor type 2 (PTPN2), T-cell activation GTPase activating protein (TAGAP), and pseudouridylate synthase 10 (PUS10), which have been reported in CeD and CD [4]. 
IBD and CeD can present with diarrhea, abdominal pain, weight loss, and poor weight gain. Various extraintestinal manifestations have been reported with the conditions, including arthritis and mouth ulcers. IBD is treated with anti-inflammatory medications, including 5-aminosalicylic acid (5-ASA) derivatives, corticosteroids, immunomodulators, and biological therapy, while CeD is treated with dietary elimination of gluten through a lifelong gluten-free diet (GFD). IBD has been associated with a variety of autoimmune disorders, including systemic lupus erythematosus (SLE), type-1 diabetes mellitus (IDDM), autoimmune hepatitis, primary sclerosing cholangitis (PSC), psoriasis, Sjögren's syndrome, and CeD [5]. The association between $\mathrm{CeD}$ and IBD has been explored in several studies, with contradictory findings [6-10]. A systematic review has shown that IBD patients have a two-fold increased risk for developing CeD [11]. Patients with IBD and concomitant $\mathrm{CeD}$ have been reported to be at higher risk of more extensive and severe disease, resulting in a greater number of hospitalisations, extensive disease involvement, and a higher association with PSC [12]. An epidemiological study of Egyptian children found a prevalence rate of CeD of at least one in 187 healthy individuals $(0.53 \%)$, and found $6.4 \%$ of $\mathrm{CeD}$ children with type 1 diabetes mellitus, but reported no studies on children with IBD [13]. There is also a lack of studies in the Middle East on CeD in children with IBD. Thus, we aim to examine the prevalence of $\mathrm{CeD}$ in a group of Egyptian children and adolescents with IBD.

\section{Materials And Methods}

This is a cross-sectional study of children aged two to 18 years with a confirmed diagnosis of IBD following up at the Pediatric Gastroenterology Unit at Cairo University Children Hospital between 2016 and 2018. The diagnosis of IBD was established based on clinical, endoscopic, histopathological, and radiological findings and after exclusion of infectious causes, immune deficiencies, and allergies. Clinical, demographic, laboratory, and radiological data were collected and analysed. Children with IBD underwent upper gastrointestinal endoscopy as part of their initial evaluation at diagnosis, and mucosal biopsies were obtained from the oesophagus, gastric antrum, gastric body, and the duodenum. Dietary history was recorded for all patients at presentation. Anthropometric data were converted into standard deviation scores (z scores) using Epi-Info software, Version 7.2 (Centers for Disease Control and Prevention, Atlanta, GA, USA), and expressed as weight-for-age z scores (WAZs), height-for-age z scores (HAZs), and body mass index (BMI) z scores.

\section{Serological screening and diagnosis of CeD}

Serological screening for CeD was performed for the study purpose by measuring the immunoglobulin A fraction of tissue transglutaminase (IgA-tTG) antibody level combined with measurement of total immunoglobulin A (IgA). The serum IgA-tTG level was determined by enzyme-linked immunosorbent assay (ELISA), using DRG ${ }^{\circledR}$, Anti-Tissue Transglutaminase (EIA-3611) (Celikey; Pharmacia Diagnostics, Freiburg, Germany). According to the manufacturer's instructions, $10 \mathrm{U} / \mathrm{mL}$ was taken as the cut off value for a positive result. Total IgA was measured using nephlometry (Nephstar, Shaanxi, China).

The diagnosis of $\mathrm{CeD}$ was established according to the revised criteria of the European Society for Pediatric Gastroenterology Hepatology and Nutrition (ESPGHAN) [14]. A positive result for IgA-tTG antibody was followed by a small intestinal biopsy examination obtained through upper gastrointestinal endoscopy, with four to six specimens taken from the second part of the duodenum. Histopathological changes were graded according to the Marsh-Oberhuber classification [15].

\section{Ethical consideration}

The study protocol was approved by the Research Ethics Committee of the Pediatrics Department, Faculty of Medicine, Cairo University, Egypt (Ref. number I-071017). The research was conducted in accordance with the Declaration of Helsinki.

\section{Statistical analysis}

The Statistical Package for the Social Sciences (SPSS), version 22 (IBM Corp., Armonk, NY, USA) was used for data analysis. Descriptive statistics were used to express data, using mean and standard deviation (SD) or median and interquartile range (IQR) for continuous data and percentages for categorical data. For group comparison, we used a t-test for continuous variables and Fisher's exact and chi-squared $(\chi 2)$ tests for categorical variables. A P value of $<0.05$ was set as statistically significant.

\section{Results}

\section{Baseline characteristics}

The present study included 101 patients with an established diagnosis of IBD: 84 patients (83.2\%) had UC and 17 patients (16.8\%) had CD. The mean age of the participants at the time of inclusion in the study was $8.7 \pm 4.0$ years and males constituted $59.4 \%(n=60)$ of the cohort. The mean duration of illness following diagnosis of IBD when screened for $\mathrm{CeD}$ was $2.4 \pm 1.8$ years. The most common disease location for $\mathrm{CD}$ patients was the ileocolonic region (64.5\%); the most common behavior was non-stricturing and nonpenetrating disease (76.7\%); a small percentage (3\%) had perianal disease. The majority of UC patients 


\section{Cureus}

(45.2\%) had pancolitis. Extraintestinal manifestations were reported in $9.9 \%$ of the total cohort. Baseline clinical and demographic characteristics are summarized in Table 1.

\begin{tabular}{|c|c|}
\hline & Number $(\%)$ or mean \pm SD \\
\hline Age at diagnosis (years) & $6.5 \pm 3.6$ \\
\hline Male gender & $60(59.4 \%)$ \\
\hline Crohn's disease (CD) & $17(16.8 \%)$ \\
\hline Ulcerative colitis (UC) & $84(83.2 \%)$ \\
\hline \multicolumn{2}{|l|}{ Montreal Classification } \\
\hline \multicolumn{2}{|l|}{ Disease location (CD) } \\
\hline L1: lleal location & $6(35.3 \%)$ \\
\hline L2: Colonic location & $0(0.0 \%)$ \\
\hline L3: Ileocolonic location & $11(64.7 \%)$ \\
\hline \multicolumn{2}{|l|}{ Disease behaviour (CD) } \\
\hline B1: Non-stricturing, non-penetrating & $13(76.5 \%)$ \\
\hline B2: Stricturing & $4(23.5 \%)$ \\
\hline B3: Penetrating & $0(0.0 \%)$ \\
\hline \multicolumn{2}{|l|}{ Disease Extension (UC) } \\
\hline E1: Proctitis & $15(17.9 \%)$ \\
\hline E2: Left sided colitis & $31(36.9 \%)$ \\
\hline E3: Pancolitis & 38 (45.2\%) \\
\hline Perianal disease & $3(3 \%)$ \\
\hline Extra intestinal manifestations (EIMs) & $10(9.9 \%)$ \\
\hline Weight for age $\mathrm{z}$ score (WAZ) & $-1.7 \pm 1.9$ \\
\hline Height for age $\mathrm{z}$ score (HAZ) & $-2.2 \pm 2.3$ \\
\hline Body Mass Index (BMI) z score & $-0.32 \pm 2.1$ \\
\hline \multicolumn{2}{|l|}{ Laboratory investigations } \\
\hline Hemoglobin (g/dL) & $9.3 \pm 1.4$ \\
\hline Platelets (K/uL) & $304.7 \pm 92.4$ \\
\hline C-reactive protein (mg/L) & $16.1 \pm 13.5$ \\
\hline Albumin (g/L) & $36.8 \pm 5.5$ \\
\hline \multicolumn{2}{|l|}{ Treatment } \\
\hline Corticosteroids & $51(50.5 \%)$ \\
\hline Mesalamine & $43(42.6 \%)$ \\
\hline Azathioprine & $58(57.4 \%)$ \\
\hline Biological therapy & $7(7 \%)$ \\
\hline History of bowel surgery & $2(2 \%)$ \\
\hline
\end{tabular}

TABLE 1: Baseline clinical, demographic, and laboratory characteristics of the study population 


\section{Cureus}

\section{Study outcomes}

Serology screening for IgA-tTG antibodies was positive in 10 patients (10\%), with a mean IgA-tTG antibody value of $126.5 \pm 40.0 \mathrm{U} / \mathrm{mL}$. Out of the 10 patients with positive serology, three patients (3\%) had IgA-tTG level more than 10 times the upper limit of normal, and seven patients (70\%) were below this limit. None of the patients had a low total IgA antibody titre. Three patients (3\%) that had a positive IgA-tTG serology underwent upper endoscopy and small bowel biopsy that showed total villous atrophy and crypt hyperplasia with increased intraepithelial lymphocytes, compatible with the diagnosis of celiac disease (Marsh III c). Two patients had CD (66.7\%) and one had UC (33.3\%). Of the patients diagnosed with CeD and UC, one had proctitis and one had left-sided colitis. The patient diagnosed with $\mathrm{CD}$ had an ileocolonic phenotype. Patients with confirmed CeD had a significantly higher frequency of symptoms of gaseous sensation and bloating ( $\mathrm{P}=0.003)$ (Table 2).

\begin{tabular}{|c|c|c|c|}
\hline & Non CeD patients $\mathrm{N}(\%)$ or mean \pm SD $\mathrm{N}=98$ & CeD patients $\mathrm{N}(\%)$ or mean $\pm \mathrm{SD} \mathrm{N}=3$ & ${ }^{*} \mathrm{P}$ value \\
\hline \multicolumn{4}{|l|}{ IBD subtypes } \\
\hline Crohn's disease (CD) & $15(15.3 \%)$ & $2(66.7 \%)$ & \multirow{2}{*}{0.07} \\
\hline Ulcerative colitis (UC) & $83(84.7 \%)$ & $1(33.3 \%)$ & \\
\hline \multicolumn{4}{|l|}{ Gender } \\
\hline Female & $41(41.8 \%)$ & $0(0.0 \%)$ & \multirow{2}{*}{0.27} \\
\hline Male & $57(58.2 \%)$ & $3(100 \%)$ & \\
\hline Age at enrollment & $8.6 \pm 4.0$ & $11.3 \pm 3.8$ & 0.26 \\
\hline \multicolumn{4}{|l|}{ Anthropometric measures } \\
\hline Weight for age $\mathrm{z}$ score & $-1.7 \pm 1.8$ & $-2.9 \pm 2.7$ & 0.27 \\
\hline Height for age $\mathrm{z}$ score & $-2.2 \pm 2.3$ & $-3.2 \pm 1.2$ & 0.45 \\
\hline Body mass index z score & $-0.30 \pm 2.1$ & $-0.95 \pm 2.3$ & 0.59 \\
\hline \multicolumn{4}{|l|}{ Clinical manifestations } \\
\hline \multicolumn{4}{|l|}{ Weight loss } \\
\hline Yes & $61(62.2 \%)$ & $3(100 \%)$ & \multirow{2}{*}{0.29} \\
\hline No & $37(37.8 \%)$ & $0(0.0 \%)$ & \\
\hline \multicolumn{4}{|l|}{ Rectal bleeding } \\
\hline Yes & $81(82.7 \%)$ & $1(33.3 \%)$ & \multirow{2}{*}{0.09} \\
\hline No & $17(17.3 \%)$ & $2(66.7 \%)$ & \\
\hline \multicolumn{4}{|l|}{ Tenesmus } \\
\hline Yes & $71(72.4 \%)$ & $1(33.3 \%)$ & \multirow{2}{*}{0.19} \\
\hline No & $27(27.6 \%)$ & $2(66.7 \%)$ & \\
\hline \multicolumn{4}{|l|}{ Abdominal pain } \\
\hline Yes & $94(95.9 \%)$ & $3(100 \%)$ & \multirow{2}{*}{1.00} \\
\hline No & $4(4.1 \%)$ & $0(0.0 \%)$ & \\
\hline \multicolumn{4}{|l|}{ Uiarrnea } \\
\hline Yes & $78(79.6 \%)$ & $3(100 \%)$ & \multirow{2}{*}{1.00} \\
\hline No & $20(20.4 \%)$ & $0(0.0 \%)$ & \\
\hline \multicolumn{4}{|l|}{ Perianal disease } \\
\hline Yes & $2(2.0 \%)$ & $1(33.3 \%)$ & \multirow{2}{*}{0.09} \\
\hline No & 96 (98\%) & 2 (66.7\%) & \\
\hline
\end{tabular}




\section{Cureus}

Yes

No

Gaseous sensation and bloating

Yes

No

Hepatomegaly

Yes

No

Splenomegaly

Yes

No

Laboratory measures

Hemoglobin (g/dL)

Platelets (K/uL)

Albumin (g/L)

C-reactive protein (mg/L)
$9(9.2 \%)$

$1(33.3 \%)$

$89(90.8 \%)$

$2(66.7 \%)$

$12(12.2 \%)$

$3(100 \%)$

$86(87.8 \%)$

$0(0.0 \%)$

$1(33.3 \%)$

$4(4.1 \%)$

$2(66.7 \%)$

0.14

$94(95.9 \%)$

$1(1.0 \%)$

$0(0.0 \%)$

$3(100.0 \%)$

97 (99\%)

$8.7 \pm 1.3$

0.41

$300 \pm 100$

0.93

$33 \pm 10$

0.27

$17.3 \pm 19.6$

0.87

TABLE 2: Bivariate analysis of inflammatory bowel disease (IBD) patients with celiac disease (CeD) and patients without CeD

*Fisher's exact or t-test

${ }^{* *} \mathrm{P}<0.05$

Including patients with elevated IgA-tTG and negative biopsy, symptoms of gaseous sensation and bloating were more frequently reported in serology-positive patients than serology-negative patients (9/10 vs. 6/91, $\mathrm{P}<0.001)$. Comparing the seroprevalence of $\mathrm{CeD}$ in our study $(10 \%)$ with the seroprevalence of $\mathrm{CeD}$ in the pediatric general population in Egypt (0.5\%), the difference was statistically significant (Fisher's exact, $\mathrm{P}<0.001)$.

\section{Clinical course following CeD diagnosis}

Patients with a confirmed diagnosis of CeD were put on a GFD. All patients reported improvement in their symptoms of abdominal pain, bloating, and distention following the implementation of a GFD. Patients with elevated IgA-tTG and negative small bowel biopsy were scheduled for annual repeat of serology and rebiopsy if the serology is still elevated.

\section{Discussion}

In this cross-sectional study, we examined the coexistence of $\mathrm{CeD}$ and IBD in a cohort of pediatric patients. Several case reports in children have described the simultaneous existence of UC and CeD, which can affect both boys and girls [16-18] and can occur in association with PSC [19]. We found an association between CeD and UC, and an association between CeD and CD in patients. The relationship between these conditions may be a result of an overlap in the dysregulated immune pathway that underlies the conditions. Polymorphisms involving the IL-21/IL23R genes have been described in cases where UC and CeD coexist [20]; in both these conditions the expression of IL-21 is increased. IL-21 influences the T cell helper 1 response in CeD and the Th17 cell proinflammatory function [3,21]. However, studies that have estimated the prevalence of CeD in children with IBD have found variable results. A recent systematic review and meta-analysis of various pediatric and adult populations reported an increased risk of $\mathrm{CeD}$ in IBD vs. control (relative risk [RR]=2.9), of $\mathrm{CeD}$ in $\mathrm{CD}$ vs. control ( $\mathrm{RR}=3.15)$, and of $\mathrm{CeD}$ in $\mathrm{UC}$ vs. control $(\mathrm{RR}=2.81)$ [22]. The prevalence of $\mathrm{CeD}$ in our study is approximately $3 \%$, which is higher than the prevalence rate of $0.53 \%$ reported for the general population of children aged seven months to 18 years in Egypt [13], and also higher than reported in a pediatric IBD study in Finland (2.2\% vs. $0.7 \%$ in the control group) [23]. In contrast, a retrospective study in New Haven, Connecticut reported no significant difference between the prevalence of CeD in children with IBD and a control group ( $0.8 \%$ vs. $4.7 \%, \mathrm{P}=0.07)$, but trending towards a greater prevalence in the controls 
[24]. In a matched birth cohort study conducted in children and young adults in the Friuli-Venezia Giulia region of Italy, it was shown that patients with CeD might have an increased risk of developing IBD (odds ratio $[\mathrm{OR}]=24.17$; 95\%CI, 10.03-58.21) [25]. According to a study also based in Italy, the reported prevalence of CeD in adult patients with IBD was 0.5\% [26]; similar studies in adults in Sweden [27], Turkey [6], and Iran [28] reported prevalence rates of $2.2 \%, 5.06 \%$, and $0.5 \%$, respectively.

In our study, we found $\mathrm{CeD}$ in two patients with $\mathrm{CD}(11.8 \%)$ and in one patient with UC (1.2\%), which is consistent with some other studies in adults [5,9]. In support of these findings, Tursi et al. found that $18.5 \%$ of patients with CD had biopsy-proven CeD [10], and Bengi et al. detected CeD in 7.5\% of CD patients and in only $2.6 \%$ of patients with UC [6].

Owing to the similarity of symptoms of CeD and IBD, CeD diagnosis in patients with IBD without serological screening may be difficult, using either IgA-tTG or endomysial antibodies. The effect of immunomodulatory therapies for IBD that also treat CeD may ameliorate symptoms and result in CeD being undetectable. Furthermore, the widespread utilisation of GFD in IBD patients may cause mucosal healing of any undiagnosed CeD. Dietary history of participants in our study excluded the confounding effect of GFD consumption by the IBD patients on small bowel mucosal healing. False-positive serological test results, with no histological changes, have been reported in a number of studies performed with adults [29,30]. Our analysis identified associations of excessive gases and bloating with $\mathrm{CeD}$; this, to our knowledge, has not been previously reported.

Our study may be limited by its retrospective design, a lack of a control group of non-IBD children, and a lack of long-term monitoring following introduction of a GFD.

\section{Conclusions}

In conclusion, our study showed a high prevalence rate of $\mathrm{CeD}$ in Egyptian children with IBD when compared to the pediatric general population. Accordingly, CeD could be considered during diagnosis in the context of additional clinical symptoms, such as the presence of excessive abdominal gaseous sensation and bloating.

\section{Additional Information \\ Disclosures}

Human subjects: Consent was obtained by all participants in this study. The Research Ethics Committee of the Pediatrics Department, Faculty of Medicine, Cairo University, Egypt issued approval Ref. number I071017. The study protocol was approved by the Research Ethics Committee of the Pediatrics Department, Faculty of Medicine, Cairo University, Egypt (Ref. number I-071017). The research was conducted in accordance with the Declaration of Helsinki. Animal subjects: All authors have confirmed that this study did not involve animal subjects or tissue. Conflicts of interest: In compliance with the ICMJE uniform disclosure form, all authors declare the following: Payment/services info: All authors have declared that no financial support was received from any organization for the submitted work. Financial relationships: All authors have declared that they have no financial relationships at present or within the previous three years with any organizations that might have an interest in the submitted work. Other relationships: All authors have declared that there are no other relationships or activities that could appear to have influenced the submitted work.

\section{Acknowledgements}

The authors acknowledge Dr. Trevor Rawbone, Cardiff, UK, for English editing and proofreading of the manuscript.

\section{References}

1. Lamb CA, Kennedy NA, Raine T, et al.: British Society of Gastroenterology consensus guidelines on the management of inflammatory bowel disease in adults. Gut. 2019, 68:1-106. 10.1136/gutjnl-2019-318484

2. Guandalini S: The approach to celiac disease in children . Int J Pediatr Adolesc Med. 2017, 4:124-7. 10.1016/j.ijpam.2017.08.002

3. Pascual V, Dieli-Crimi R, Lopez-Palacios N, Bodas A, Medrano LM, Nunez C: Inflammatory bowel disease and celiac disease: overlaps and differences. World J Gastroenterol. 2014, 20:4846-56. 10.3748/wjg.v20.i17.4846

4. Festen EA, Goyette P, Green T, et al.: A meta-analysis of genome-wide association scans identifies IL18RAP, PTPN2, TAGAP, and PUS10 as shared risk loci for Crohn's disease and celiac disease. PLoS Genet. 2011, 7:1001283. 10.1371/journal.pgen.1001283

5. Bar Yehuda S, Axlerod R, Toker O, et al.: The association of inflammatory bowel diseases with autoimmune disorders: a report from the epi-IIRN. J Crohns Colitis. 2019, 13:324-9. 10.1093/ecco-jcc/jjy166

6. Bengi G, Civak M, Akarsu M, et al.: Prevalance of celiac disease in patients with inflammatory bowel disease in Turkish population. Gastroenterol Res Pract. 2019, 2019:6272098. 10.1155/2019/6272098

7. Culliford A, Markowitz D, Rotterdam H, Green PH: Scalloping of duodenal mucosa in Crohn's disease . Inflamm Bowel Dis. 2004, 10:270-3. 10.1097/00054725-200405000-00015 
8. Leeds JS, Horoldt BS, Sidhu R, et al.: Is there an association between coeliac disease and inflammatory bowel diseases? A study of relative prevalence in comparison with population controls. Scand J Gastroenterol. 2007, 42:1214-20. 10.1080/00365520701365112

9. Targan SR, Karp LC: Inflammatory bowel disease diagnosis, evaluation and classification: state-of-the art approach. Curr Opin Gastroenterol. 2007, 23:390-4. 10.1097/MOG.0b013e3281722271

10. Tursi A, Giorgetti GM, Brandimarte G, Elisei W: High prevalence of celiac disease among patients affected by Crohn's disease. Inflamm Bowel Dis. 2005, 11:662-6. 10.1097/01.mib.0000164195.75207.1e

11. Shah A, Walker M, Burger D, et al.: Link between celiac disease and inflammatory bowel disease . J Clin Gastroenterol. 2019, 53:514-522. 10.1097/MCG.0000000000001033

12. Tse CS, Deepak P, De La Fuente J, et al.: Phenotype and clinical course of inflammatory bowel disease with co-existent celiac disease. J Crohns Colitis. 2018, 12:973-80. 10.1093/ecco-jcc/jjy061

13. Abu-Zekry M, Kryszak D, Diab M, Catassi C, Fasano A: Prevalence of celiac disease in Egyptian children disputes the east-west agriculture-dependent spread of the disease. J Pediatr Gastroenterol Nutr. 2008, 47:136-40. 10.1097/MPG.0b013e31815ce5d1

14. Husby S, Koletzko S, Korponay-Szabo IR, et al.: European Society for Pediatric Gastroenterology, Hepatology, and Nutrition guidelines for the diagnosis of coeliac disease. J Pediatr Gastroenterol Nutr. 2012, 54:136-60. 10.1097/MPG.0b013e31821a23d0

15. Marsh MN, Johnson MW, Rostami K: Rebutting Oberhuber- again. Gastroenterol Hepatol Bed Bench. 2015, 8:303-5. 10.22037/ghfbb.v8i4.799

16. Cheng SX, Raizner A, Phatak UP, Cho JH, Pashankar DS: Celiac disease in a child with ulcerative colitis: a possible genetic association. J Clin Gastroenterol. 2013, 47:127-9. 10.1097/MCG.0b013e318250e468

17. Day AS, Abbott GD: Simultaneous presentation of coeliac disease and ulcerative colitis in a child . J Paediatr Child Health. 1999, 35:204-6. 10.1046/j.1440-1754.1999.t01-1-00328.x

18. Oxford EC, Nguyen DD, Sauk J, et al.: Impact of coexistent celiac disease on phenotype and natural history of inflammatory bowel diseases. Am J Gastroenterol. 2013, 108:1123-9. 10.1038/ajg.2013.20

19. Cadahia V, Rodrigo L, Fuentes D, Riestra S, de Francisco R, Fernandez M: Celiac disease (CD), ulcerative colitis (UC), and primary sclerosing cholangitis (PSC) in one patient: a family study. Rev Esp Enferm Dig. 2005, 97:907-13. 10.4321/s1130-01082005001200007

20. Glas J, Stallhofer J, Ripke S, et al.: Novel genetic risk markers for ulcerative colitis in the IL2/IL21 region are in epistasis with IL23R and suggest a common genetic background for ulcerative colitis and celiac disease. Am J Gastroenterol. 2009, 104:1737-44. 10.1038/ajg.2009.163

21. Fina D, Sarra M, Caruso R, et al.: Interleukin 21 contributes to the mucosal T helper cell type 1 response in coeliac disease. Gut. 2008, 57:887-92. 10.1136/gut.2007.129882

22. Pinto-Sanchez MI, Seiler CL, Santesso N, et al.: Association between inflammatory bowel diseases and celiac disease: a systematic review and meta-analysis. Gastroenterology. 2020, 10.1053/j.gastro.2020.05.016

23. Virta LJ, Kolho KL: The risk of contracting pediatric inflammatory bowel disease in children with celiac disease, epilepsy, juvenile arthritis and type 1 diabetes--a nationwide study. J Crohns Colitis. 2013, 7:53-7. 10.1016/j.crohns.2012.02.021

24. Alper A, Rojas-Velasquez D, Pashankar DS: Prevalence of anti-tissue transglutaminase antibodies and celiac disease in children with inflammatory bowel disease. J Pediatr Gastroenterol Nutr. 2018, 66:934-6. 10.1097/MPG.0000000000001877

25. Canova C, Pitter G, Zanier L, Zanotti R, Simonato L, Ludvigsson JF: Inflammatory bowel diseases in children and young adults with celiac disease. A multigroup matched comparison. Inflamm Bowel Dis. 2017, 23:1996-2000. 10.1097/MiB.0000000000001098

26. Casella G, D'Inca R, Oliva L, et al.: Prevalence of celiac disease in inflammatory bowel diseases: an IG-IBD multicentre study. Dig Liver Dis. 2010, 42:175-8. 10.1016/j.dld.2009.08.005

27. Ronnblom A, Holmstrom T, Tanghoj H, Wanders A, Sjoberg D: Celiac disease, collagenous sprue and microscopic colitis in IBD. Observations from a population-based cohort of IBD (ICURE). Scand J Gastroenterol. 2015, 50:1234-40. 10.3109/00365521.2015.1041152

28. Jandaghi E, Hojatnia M, Vahedi H, Shahbaz-Khani B, Kolahdoozan S, Ansari R: Is the prevalence of celiac disease higher than the general population in inflammatory bowel diseaese?. Middle East J Dig Dis. 2015, $7: 82-7$.

29. Di Tola M, Sabbatella L, Anania MC, et al.: Anti-tissue transglutaminase antibodies in inflammatory bowel disease: new evidence. Clin Chem Lab Med. 2004, 42:1092-7. 10.1515/CCLM.2004.225

30. Watanabe C, Komoto S, Hokari R, et al.: Prevalence of serum celiac antibody in patients with IBD in Japan . J Gastroenterol. 2014, 49:825-34. 10.1007/s00535-013-0838-6 